\title{
THE WICIE BIRD RINGING STATION (N POLAND) - RINGING RESULTS AND SEASONAL BIRD MIGRATION DYNAMICS IN 2010-2014
}

\author{
Grzegorz Zaniewicz, Katarzyna Rosińska
}

\begin{abstract}
Zaniewicz G., Rosińska K. 2015. The Wicie bird ringing station (N Poland) - ringing results and seasonal bird migration dynamics in 2010-2014. Ring 37, 1: 55-64.

The Wicie ringing station was one of several stations which cooperate within the SEEN organization (SE European Bird Migration Network). The station was located west of the small village of Wicie located in northern Poland on the central part of the Baltic Sea coast. The station was situated on a narrow spit between Kopań Lake and the Baltic Sea and has operated since 2010. Data were collected during three spring and five autumn seasons. Birds were caught in mist-nets, which were placed mainly in bushes and reed beds. Over 55000 birds of 113 species were caught and ringed during eight migratory seasons. Many of them were also tested for directional preferences in Busse's cages.
\end{abstract}

G. Zaniewicz (zidia@wp.pl), K. Rosińska, Bird Migration Research Foundation, Przebendowo 3, 84-210 Choczewo, Poland

Publication appointed to the SE European Bird Migration Network papers

Keywords: ringing, bird migration, Kopań Lake

\section{STUDY AREA AND METHODS}

The Wicie ringing station $\left(54^{\circ} 29^{\prime} \mathrm{N}, 16^{\circ} 27^{\prime} \mathrm{E}\right)$ was situated in northern Poland, precisely in the middle of the Polish Baltic Sea coast, $262 \mathrm{~km}$ from the eastern border. The camp was located on a narrow spit of land (700 m wide) between the Baltic Sea and Lake Kopań. The habitat where the nets were placed was variable. Most of them were set on a wet meadow surrounded by a mixed forest consisting mainly of Common Alder (Alnus glutinosa), but also Oak (Quercus robur), Birch (Betula pendula) and Pine (Pinus sylvestris), with an admixture of berry species: Elderberry (Sambucus nigra), Mountain-ash (Sorbus aucuparia), Blackthorn (Prunus spinosa), Hawthorn (Crateagus sp.) and Alder Buckthorn (Frangula alnus). The habitat is in the next stage 
of succession from peatland formation and now the area is covered by Willow (Salix sp.) and Alder Buckthorn bushes surrounded by a carpet of Soft Rush (Juncus effuses).

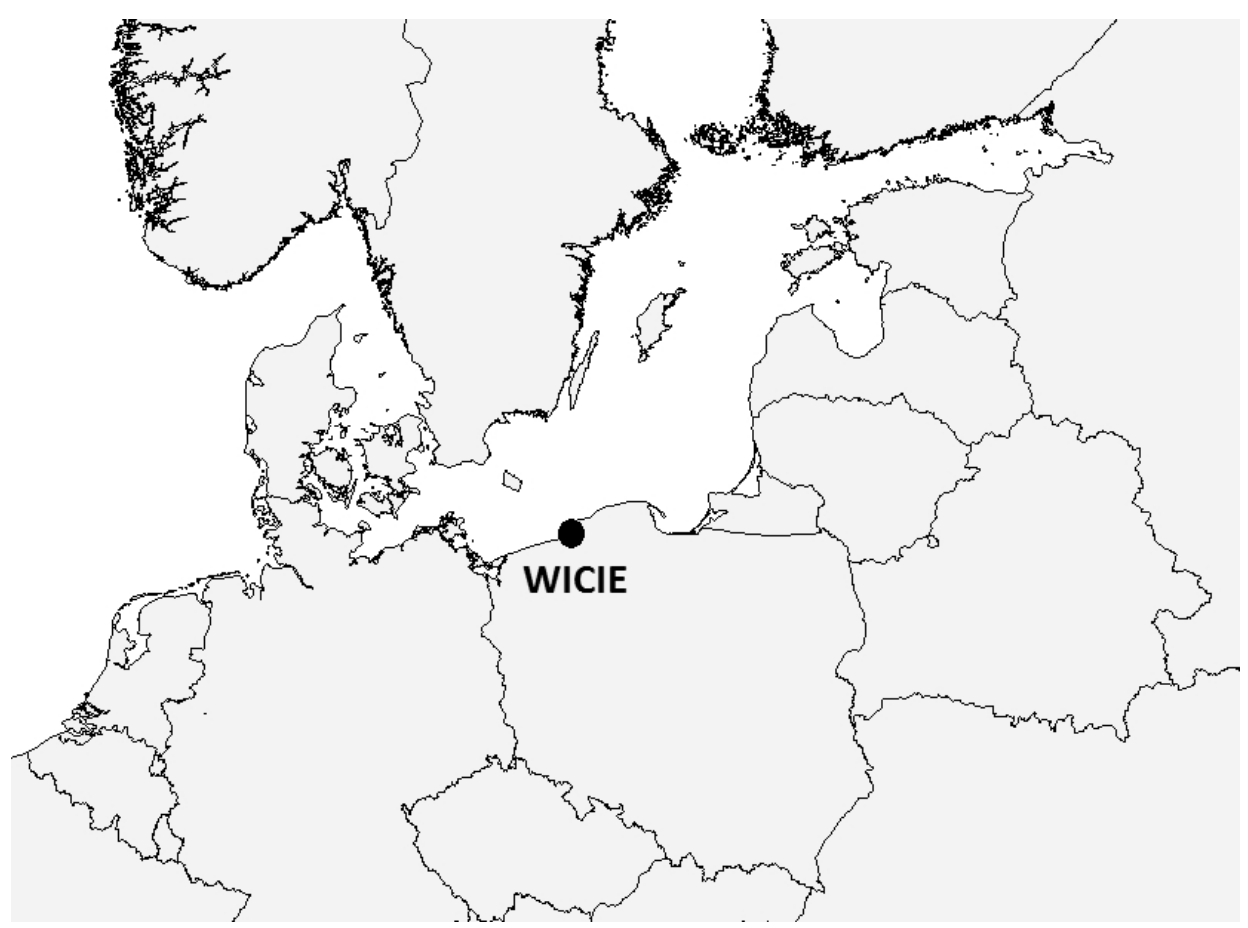

Fig. 1. Location of the Wicie ringing station

The ringing station was manned in the years 2010-2014, but netting covered nearly the entire spring and autumn bird migration seasons only between 2011 and 2013. Spring activities began on 25 March and continued to 25 May. During the autumn season netting was conducted from the beginning of August to midNovember. All nets were checked every hour from dusk to dawn. During all seasons we used about 450 linear metres of nets with mesh size $16 \mathrm{~mm}$, usually placed in the same locations. During the field work we followed the standard protocol used by Operation Baltic and the SEEN network (Busse and Meissner 2015).

After species determination the birds caught were aged and sexed (if possible) according to plumage features and sometimes skull ossification (Busse 1984, Svensson 1992), ringed, and measured. The data determined in the trapped birds included the level of fat deposits on a 9-point scale (from 0 to 8) proposed by Busse (2000), wing and tail length, wing formula, and weight to the nearest $0.1 \mathrm{~g}$. Moreover, some of nocturnal migrants were tested in Busse's cages for their directional preferences (Busse 1995, 2000; Busse and Meissner 2015). 


\section{RESULTS}

During three spring and five autumn seasons 55264 birds of 113 species were caught and ringed. During spring migration 14397 birds were ringed, and over twice this number in autumn - 40 867. In eight seasons of activity the Wicie ringing station provided 24 records of rare species, verified by the Polish Rarities Committee (Table 1).

Table 1

Number of ringed individuals of a particular species.

Species printed in bold are rare in Poland.

\begin{tabular}{|c|c|c|c|c|c|c|c|c|c|}
\hline \multirow{2}{*}{ Scientific name } & \multicolumn{3}{|c|}{ Spring } & \multicolumn{5}{|c|}{ Autumn } & \multirow[t]{2}{*}{ Total } \\
\hline & 2010 & 2011 & 2013 & 2010 & 2011 & 2012 & 2013 & 2014 & \\
\hline Acanthis hornemanni & & & & 2 & & & & & 2 \\
\hline Acanthis flammea & 8 & 5 & 6 & 163 & 6 & 2 & 32 & 5 & 227 \\
\hline Accipiter nisus & 2 & & 1 & 9 & 5 & 7 & 16 & 7 & 47 \\
\hline Acrocephalus arundinaceus & 13 & 3 & 1 & & 4 & 13 & 1 & 15 & 50 \\
\hline Acrocephalus dumetorum & & 1 & 1 & & & & & & 2 \\
\hline Acrocephalus palustris & 3 & 8 & 15 & 2 & 58 & 85 & 29 & 82 & 282 \\
\hline Acrocephalus schoenobaenus & 51 & 8 & 8 & 2 & 19 & 8 & 11 & 13 & 120 \\
\hline Acrocephalus scirpaceus & 124 & 22 & 10 & 16 & 202 & 277 & 76 & 388 & 1115 \\
\hline Aegithalos caudatus & 14 & 2 & 8 & 221 & 29 & 54 & 26 & 69 & 423 \\
\hline Aegolius funereus & & & & 1 & 1 & 1 & & 5 & 8 \\
\hline Alcedo atthis & & 2 & & & 2 & 4 & & & 8 \\
\hline Anthus pratensis & & & & 2 & 2 & & & 2 & 6 \\
\hline Anthus trivialis & 4 & 4 & 4 & 10 & 7 & 4 & 3 & 3 & 39 \\
\hline Alauda arvensis & & & & & 1 & & & & 1 \\
\hline Apus apus & 1 & & & & & & & & 1 \\
\hline Asio fllammeus & & & & 5 & 6 & 16 & 5 & 12 & 44 \\
\hline Asio otus & & & 1 & 34 & 38 & 18 & 28 & 30 & 149 \\
\hline Bombycilla garrulus & & & & & & 7 & & & 7 \\
\hline Buteo buteo & & & & & 2 & & & & 2 \\
\hline Caprimulgus europaeus & & & & 1 & 1 & 1 & & 2 & 5 \\
\hline Carduelis carduelis & 3 & 2 & & & 1 & 2 & & & 8 \\
\hline Certhia brahydactyla & 1 & 1 & 4 & 7 & & 4 & 3 & 4 & 24 \\
\hline Certhia familiaris & 21 & 4 & 12 & 75 & 13 & 39 & 25 & 90 & 279 \\
\hline Chloris chloris & 5 & 3 & 1 & & 1 & 1 & 2 & 1 & 14 \\
\hline Chroicocephalus ridibundus & & & & & 1 & & & & 1 \\
\hline Coccothraustes coccothraustes & 1 & 6 & 4 & & 1 & 2 & 3 & 1 & 18 \\
\hline Columba palumbus & 1 & & & & 1 & 1 & 1 & & 4 \\
\hline Cuculus canorus & & & & & 2 & 1 & & 3 & 6 \\
\hline Cyanistes caerulus & 44 & 12 & 78 & 285 & 330 & 397 & 173 & 136 & 1455 \\
\hline Delichon urbica & & & & & 4 & & & & 4 \\
\hline Dendrocopos major & 5 & 3 & 8 & 18 & 6 & 20 & 18 & 8 & 86 \\
\hline
\end{tabular}




\begin{tabular}{|c|c|c|c|c|c|c|c|c|c|}
\hline \multirow{2}{*}{ Scientific name } & \multicolumn{3}{|c|}{ Spring } & \multicolumn{5}{|c|}{ Autumn } & \multirow[t]{2}{*}{ Total } \\
\hline & 2010 & 2011 & 2013 & 2010 & 2011 & 2012 & 2013 & 2014 & \\
\hline Dendrocopos medius & & & & 2 & 1 & 2 & 11 & & 16 \\
\hline Dendrocopus minor & & & & 3 & 2 & 5 & 3 & 2 & 15 \\
\hline Dryocopos martius & & & & 1 & 1 & & 1 & 1 & 4 \\
\hline Emberiza citrinella & 2 & 4 & 10 & 5 & 7 & 3 & 20 & 9 & 60 \\
\hline Emberiza hortulana & & & 1 & & & & & & 1 \\
\hline Emberiza schoeniclus & 48 & 16 & 8 & 4 & 35 & 6 & 3 & 8 & 128 \\
\hline Erithacus rubecula & 2480 & 2567 & 1308 & 2736 & 1815 & 2391 & 1721 & 2786 & 17804 \\
\hline Erythrina erythrina & 12 & 17 & 11 & & 4 & 1 & 8 & 12 & 65 \\
\hline Falco subbuteo & & & & & & & & 2 & 2 \\
\hline Ficedula albicollis & & & 1 & & 4 & 1 & & & 6 \\
\hline Ficedula hypoleuca & 87 & 51 & 98 & 17 & 124 & 72 & 85 & 68 & 602 \\
\hline Ficedula parva & 16 & 25 & 41 & 1 & 5 & 3 & 25 & 5 & 121 \\
\hline Fringilla coelebs & 113 & 104 & 167 & 98 & 94 & 95 & 52 & 63 & 786 \\
\hline Fringilla montifringilla & 1 & 1 & 3 & 4 & 3 & 6 & 1 & 9 & 28 \\
\hline Gallinago gallinago & & & & 1 & 4 & 6 & & & 11 \\
\hline Garrulus glandarius & 3 & 1 & 1 & 5 & 6 & 6 & 6 & 4 & 32 \\
\hline Hippolais icterina & 7 & 18 & 10 & & 15 & 17 & 32 & 20 & 119 \\
\hline Hirundo rustica & 10 & 2 & 2 & & 8 & 7 & 8 & 2 & 39 \\
\hline Jynx torquilla & 4 & 2 & 1 & & 3 & 3 & 7 & 3 & 23 \\
\hline Lanius collurio & 10 & 43 & 49 & 6 & 48 & 48 & 32 & 62 & 298 \\
\hline Lanius exubitor & 1 & & & 1 & 6 & 1 & 2 & & 11 \\
\hline Locustella fluviatilis & 2 & 1 & 2 & & 2 & 1 & 1 & 3 & 12 \\
\hline Locustella luscinioides & 6 & 2 & 3 & & 6 & 2 & & 7 & 26 \\
\hline Locustella naevia & 14 & 6 & 13 & 5 & 22 & 29 & 16 & 7 & 112 \\
\hline Lophophanes cristatus & 2 & 3 & & 1 & 7 & 6 & 3 & 2 & 24 \\
\hline Loxia curvirostra & & 1 & & 21 & & 5 & & & 27 \\
\hline Luscinia megarhynchos & & & & & 1 & & & & 1 \\
\hline Luscinia luscinia & 4 & 7 & 4 & & 6 & 5 & & 7 & 33 \\
\hline Luscinia svecica & 5 & 3 & & & & 2 & & & 10 \\
\hline Lymnocryptes minimus & & & & 1 & 1 & 1 & & & 3 \\
\hline Motacilla alba & 2 & 2 & 3 & & 3 & 1 & & & 11 \\
\hline Motacilla flava & & 1 & & & 1 & & & & 2 \\
\hline Muscicapa striata & 13 & 19 & 26 & 25 & 29 & 12 & 23 & 29 & 176 \\
\hline Numenius arquata & & & & & 1 & & & & 1 \\
\hline Oenanthe oenanthe & 2 & & & & 1 & 2 & & 1 & 6 \\
\hline Oriolus oriolus & & & & & & & & 3 & 3 \\
\hline Panurus biarmicus & & & & & & & & 7 & 7 \\
\hline Periparus ater & 6 & 6 & 5 & 29 & 28 & 7 & 8 & 15 & 104 \\
\hline Parus major & 102 & 20 & 336 & 733 & 574 & 2518 & 2336 & 782 & 7401 \\
\hline Phoenicurus ochruros & 2 & 1 & 5 & & 2 & 2 & 2 & 1 & 15 \\
\hline Phoenicurus phoenicurus & 104 & 70 & 106 & 58 & 63 & 38 & 70 & 53 & 562 \\
\hline Phylloscopus collybita & 135 & 97 & 104 & 49 & 107 & 89 & 67 & 82 & 730 \\
\hline Phylloscopus tristis & & & & 1 & & & & & 1 \\
\hline
\end{tabular}




\begin{tabular}{|c|c|c|c|c|c|c|c|c|c|}
\hline \multirow{2}{*}{ Scientific name } & \multicolumn{3}{|c|}{ Spring } & \multicolumn{5}{|c|}{ Autumn } & \multirow[t]{2}{*}{ Total } \\
\hline & 2010 & 2011 & 2013 & 2010 & 2011 & 2012 & 2013 & 2014 & \\
\hline Phylloscopus trochiloides & 1 & 1 & & 1 & & & & & 3 \\
\hline Phylloscopus fuscatus & & & & & 1 & & & & 1 \\
\hline Phylloscopus inornatus & & & & & 1 & 2 & 4 & 3 & 10 \\
\hline Phylloscopus proregulus & & & & 1 & & 1 & 1 & & 3 \\
\hline Phylloscopus sibilatrix & 31 & 30 & 39 & 1 & 6 & 5 & 16 & 3 & 131 \\
\hline Phylloscopus schwarzi & & & & 1 & & & & & 1 \\
\hline Phylloscopus trochilus & 113 & 110 & 81 & 24 & 207 & 127 & 91 & 84 & 837 \\
\hline Poecile montanus & 2 & 1 & 1 & 42 & 24 & 54 & 15 & 21 & 160 \\
\hline Poecile palustris & 2 & & 1 & 18 & 7 & 28 & 14 & 8 & 78 \\
\hline Porzana porzana & & & & & 1 & & & & 1 \\
\hline Pica pica & & 1 & & & & & & & 1 \\
\hline Prunella modularis & 37 & 13 & 50 & 105 & 59 & 107 & 18 & 72 & 461 \\
\hline Pyrrhula pyrrhula & 27 & 7 & 11 & 246 & 47 & 38 & 67 & 7 & 450 \\
\hline Rallus aquaticus & & & & 1 & & 4 & & & $\mathbf{5}$ \\
\hline Regulus ignicapillus & 26 & 29 & 18 & 7 & 17 & 16 & 14 & 6 & 133 \\
\hline Regulus regulus & 209 & 119 & 144 & 842 & 177 & 1119 & 61 & 1574 & 4245 \\
\hline Remiz pendulinus & 1 & 1 & 1 & & & & & & 3 \\
\hline Riparia riparia & & & & & & & & 1 & 1 \\
\hline Saxicola rubetra & 1 & & & 1 & 1 & & 1 & & 4 \\
\hline Saxicola torquata & 4 & 1 & 2 & & 13 & 6 & 4 & & 30 \\
\hline Scolopax rusticola & 1 & & & 12 & 23 & 11 & 7 & 10 & 64 \\
\hline Serinus serinus & & & & & & 1 & & & 1 \\
\hline Sitta europaea & 1 & 1 & 3 & 13 & 4 & 18 & 9 & 8 & 57 \\
\hline Spinus spinus & 134 & 100 & 550 & 938 & 116 & 463 & 116 & 120 & 2537 \\
\hline Strix aluco & & & & 2 & & 3 & 1 & 3 & 9 \\
\hline Sturnus vulgaris & & & & 3 & 19 & 8 & 82 & 118 & 230 \\
\hline Surnia ulula & & & & & & & 1 & & $\underline{1}$ \\
\hline Sylvia atricapilla & 334 & 376 & 528 & 338 & 802 & 708 & 611 & 643 & 4340 \\
\hline Sylvia borin & 52 & 61 & 82 & 42 & 227 & 150 & 195 & 195 & 1004 \\
\hline Sylvia communis & 55 & 33 & 92 & 1 & 77 & 65 & 98 & 60 & 481 \\
\hline Sylvia curruca & 197 & 134 & 136 & 11 & 76 & 85 & 80 & 50 & 769 \\
\hline Sylvia nisoria & 3 & 5 & 5 & & 19 & 11 & 11 & 33 & 87 \\
\hline Tarsiger cyanurus & & & & & & 1 & & & 1 \\
\hline Troglodytes troglodytes & 38 & 25 & 54 & 100 & 70 & 106 & 51 & 67 & 511 \\
\hline Turdus iliacus & 42 & 24 & 9 & 27 & 31 & 15 & 19 & 25 & 192 \\
\hline Turdus merula & 190 & 126 & 377 & 200 & 292 & 410 & 477 & 319 & 2391 \\
\hline Turdus philomelos & 148 & 117 & 95 & 334 & 443 & 478 & 228 & 274 & 2117 \\
\hline Turdus pilaris & & & & 11 & 8 & 3 & 2 & 6 & 30 \\
\hline Turdus torquatus & & & 1 & & & 1 & & & 2 \\
\hline Turdus viscivorus & 2 & & 1 & & 1 & & 1 & 1 & 6 \\
\hline Tyto alba & & & & 1 & & 1 & 1 & & 3 \\
\hline Total & 5145 & 4491 & 4761 & 7981 & 6551 & 10402 & 7291 & 8642 & 55264 \\
\hline
\end{tabular}


To show real dynamics of migration only individuals trapped in 2011-2013 within a standardized period were used. The standardized period for spring covered 61 days (24 March - 25 May), while for autumn it was extended to 104 days (1 August 15 November). The median date for spring migration was 18 April and for autumn 8 October (Fig. 2).
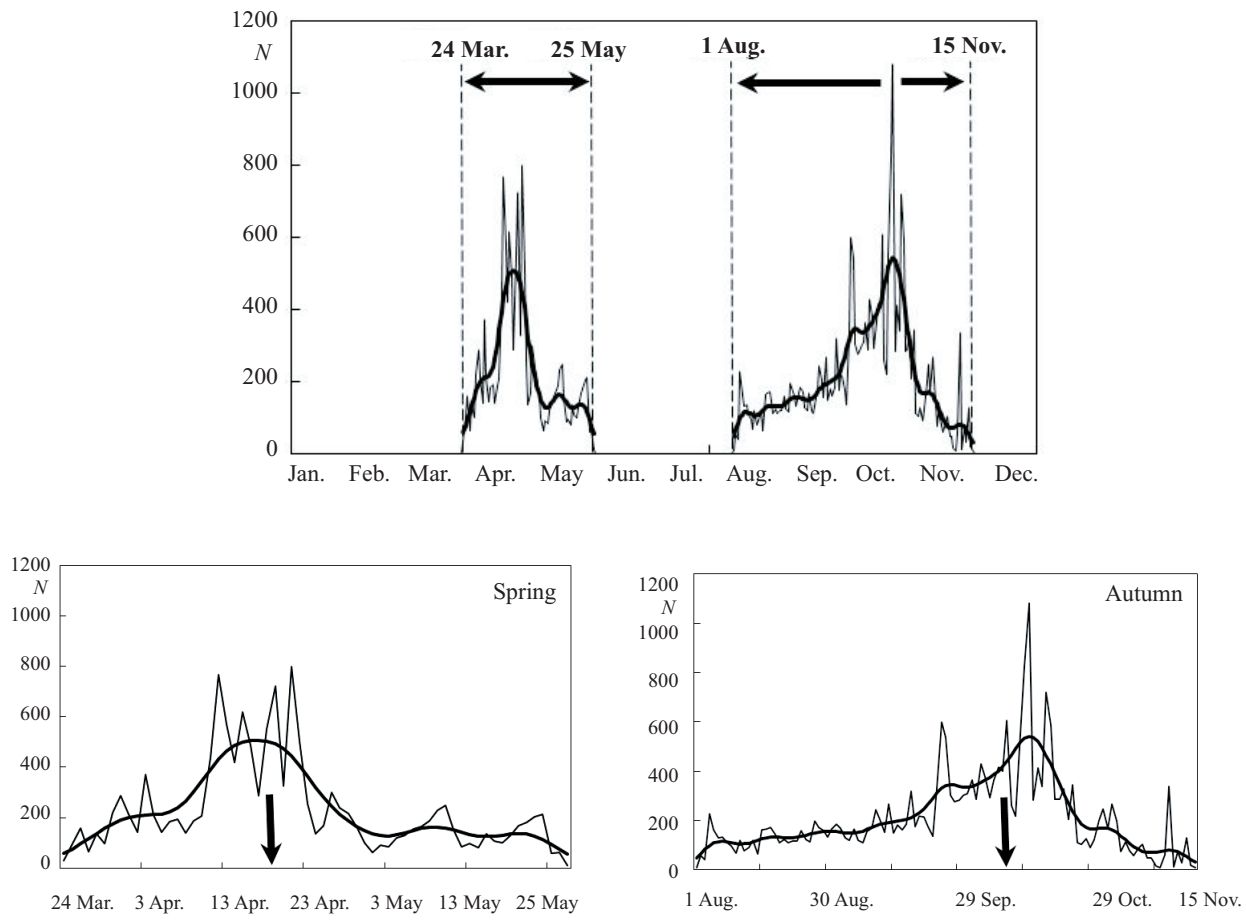

Fig. 2. Total dynamics of all birds caught at the Wicie station in standard periods in 2011-2013. Thin lines - raw daily numbers, thick lines - daily numbers smoothed by moving average. Above - general year-round pattern. Vertical broken lines show borders of standard periods. Below - seasonal results. Vertical arrows - median dates of migration in seasons.

Among the six most numerous species caught in the years 2011 to 2013, during spring migration the Siskin (Spinus spinus) was the earliest and the Blackcap (Sylvia atricapilla) the latest, while in autumn the first species to depart was the Blackcap and last were the Great Tit (Parus major) and Blackbird (Turdus merula) (Table 2).

Such an extended period of field work at $54^{\circ}$ latitude should cover most of the spring and autumn passage of passerine species, with few exceptions, such as the Common Rosefinch (Erythrina erythrin) or Siskin - very late and very early spring migrants, respectively (Fig. 3). The Siskin, as a short-distance migrant feeding mainly on seeds, can afford such an early arrival in spring. The same strategy would be highly risky for many insectivorous species, which arrive much later in the season after vegetation has fully developed. 
Table 2

Median passage date of six most numerous species caught at Wicie Station

\begin{tabular}{|l|c|c|}
\hline & Spring & Autumn \\
\hline Blackcap (Sylvia atricapilla) & 30 April & 8 September \\
\hline Robin (Erithacus rubecula) & 18 April & 29 September \\
\hline Blackbird (Turdus merula) & 11 April & 16 October \\
\hline Goldcrest (Regulus regulus) & 10 April & 14 October \\
\hline Great Tit (Parus major) & 9 April & 16 October \\
\hline Siskin (Spinus spinus) & 31 March & 10 October \\
\hline
\end{tabular}
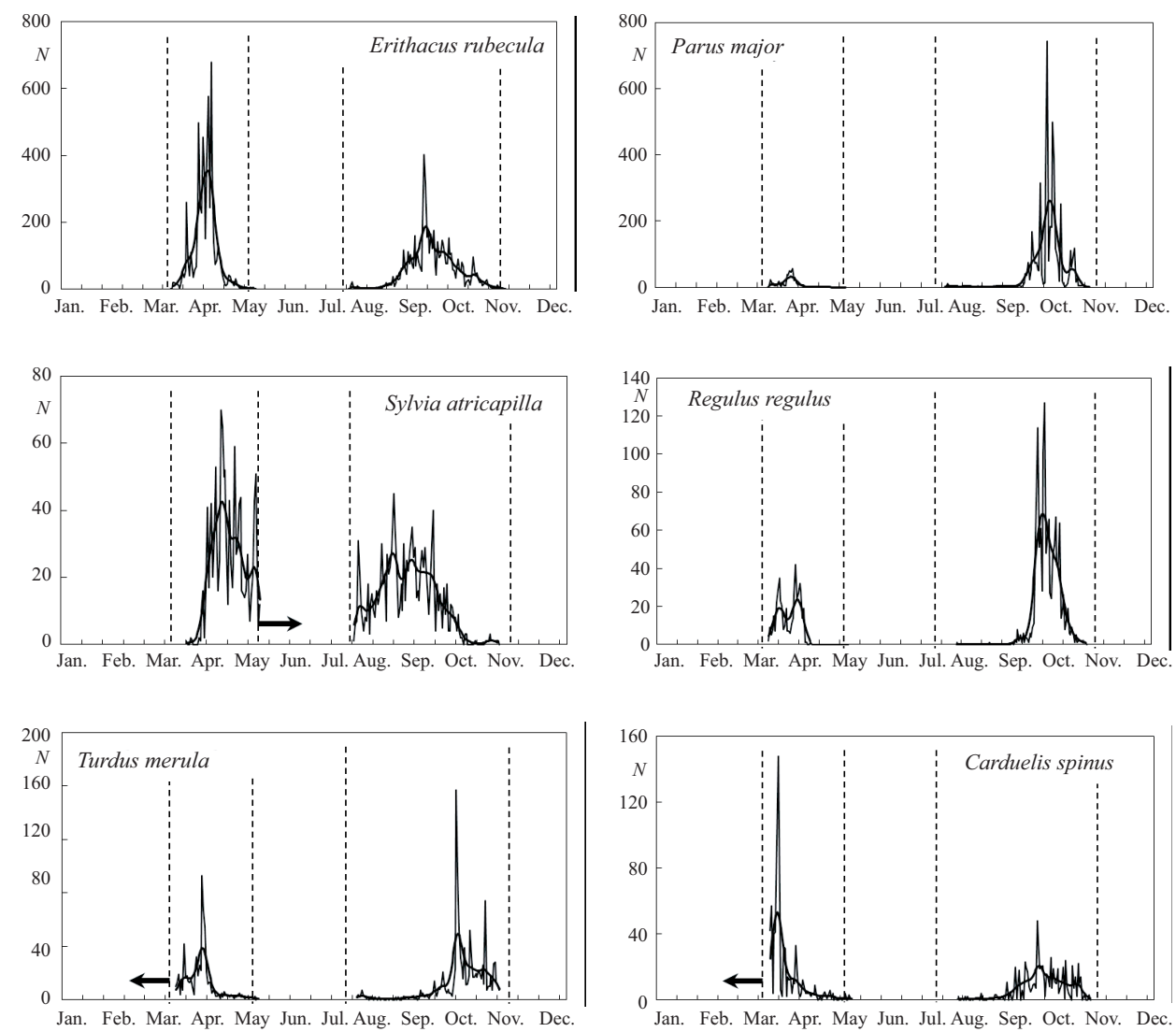

Fig. 3. Total dynamics of the six most numerous species caught at the Wicie station in standard periods in 2011-2013. Horizontal arrows indicate where the standard period may not cover the entire migration of the species. Other explanations as for Fig. 2.

The pattern of median passage dates among the six species analysed more or less followed general rules, where the latest species in spring migrate earliest in autumn (Buskirk et al. 2008). Moreover, for different species this general migration schedule is connected with distance of migration and preferred food type. A good example which confirms this mechanism is the Blackcap and the Robin (Erithacus rubecula). 

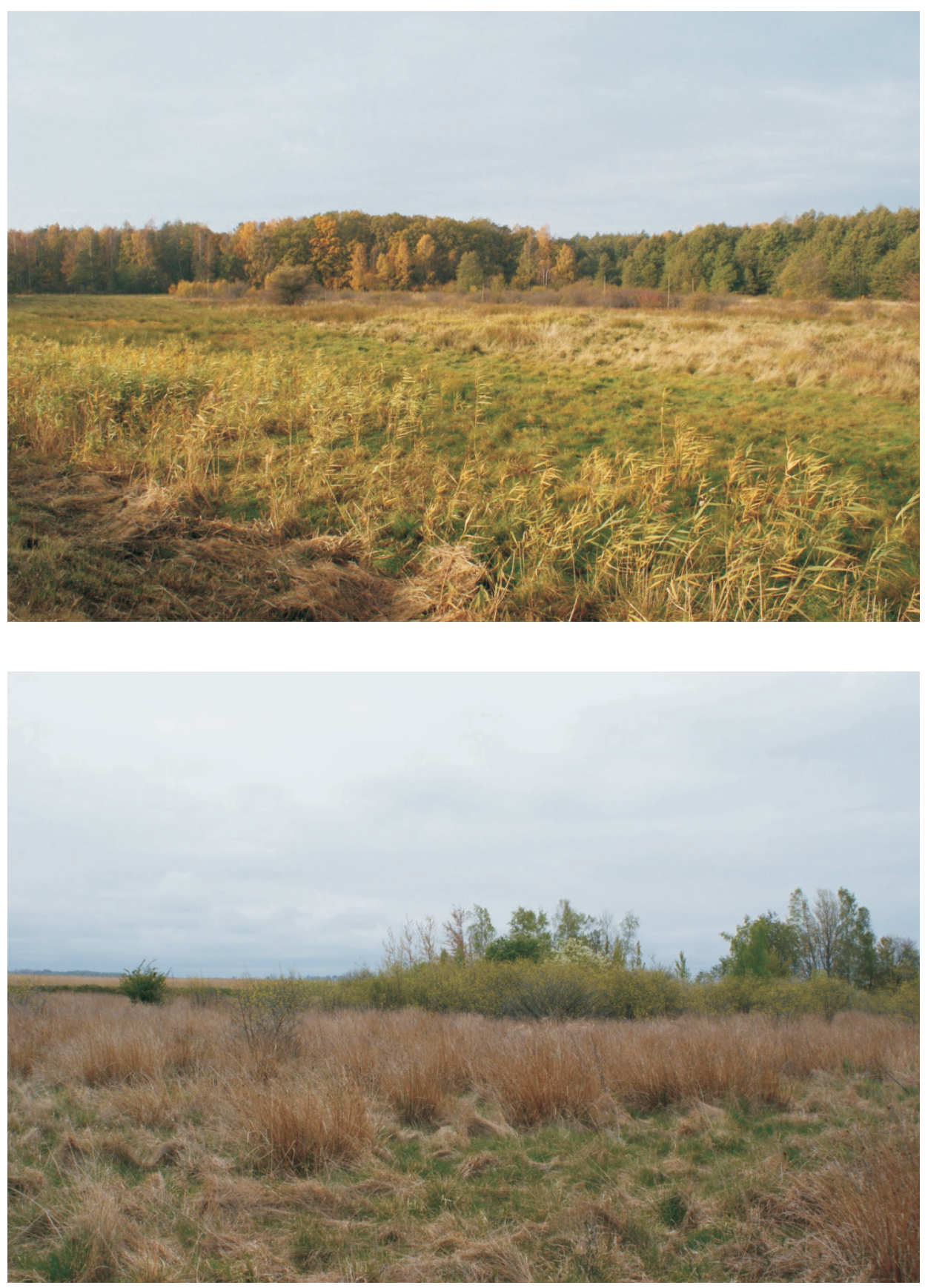

Plate 1. Biotopes surrounding the Wicie ringing station where mist-nets were placed (Photo by G. Zaniewicz) 
Both of these species are mainly insectivorous and among the species analysed they have the longest migration distance, particularly the Blackcap, of which some populations spend the winter south of the Sahara.

In the period 2011-2013, both during the spring and autumn migration seasons, the most numerous species caught was the Robin $(N$ Spring $=6355, N$ Autumn $=$ 5 927) (Fig. 4).
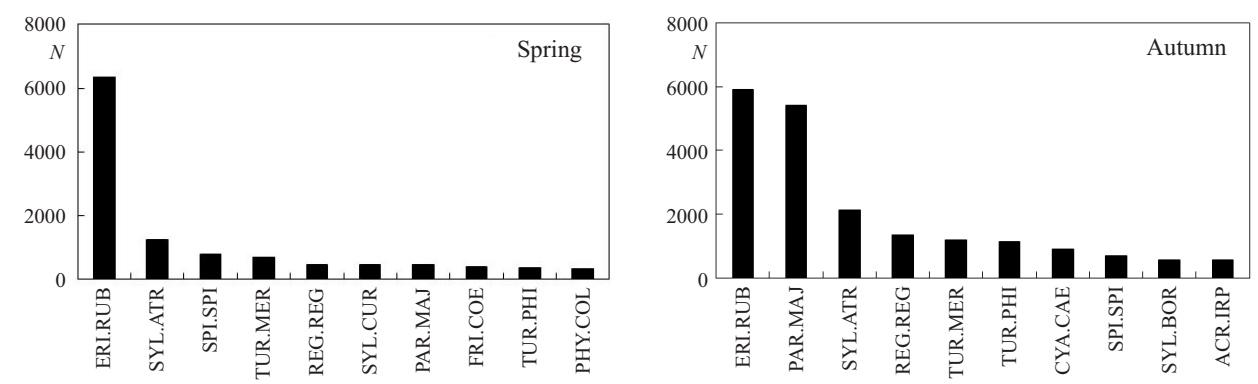

Fig. 4. Total numbers of individuals of the ten most numerous species caught at the Wicie station. ERI.RUB - Erithacus rubecula, SYL.ATR - Sylvia atricapilla, SPI.SPI - Carduelis spinus, TUR.MER - Turdus merula, REG.REG - Regulus regulus, SYL.CUR - Sylvia curruca, PAR.MAJ - Parus major, FRI.COE - Fringilla coelebs, TUR.PHI - Turdus philomelos, PHY.COL - Phylloscopus collybita, CYA.CAE - Cyanistes caeruleus, SYL.BOR - Sylvia borin, ACR.IRP - Acrocephalus scirpaceus.

\section{ACKNOWLEDGEMENTS}

We would like to thank all ringers (Przemysław Busse, Monika Filar, Małgorzata Ginter, Dariusz Górecki, Robert Lasecki, Michał Kizielewnicz, Ewelina Majchrzak, Ludwik Maksalon, Mateusz Materek, Łukasz Matyjasiak, Piotr Nagórski, Paweł Sieradzki, Sławomir Springer, Katarzyna Stępniewska, Krzysztof Stępniewski, Anna Szefler, Agnieszka Ożarowska, Halina Pietrykowska, Piotr Piliczewski and Maciej Wayda) and volunteers who were involved in the work at the Wicie ringing station. We are extremely grateful to the Board of the Bird Migration Research Foundation and to Mr. and Mrs. Hinc, representatives of the local community, for their help in solving logistical problems.

\section{REFERENCES}

Van Buskirk J., Mulvihill R. S., \& Leberman R. C. 2009. Variable shifts in spring and autumn migration phenology in North American songbirds associated with climate change. Global Change Biology 15, 3: 760-771.

Busse P. 1984. Key to sexing and ageing of European passerines. Beitr. Naturk. Niedersachsens 37, Suppl.

Busse P. 1995. New technique of a field study of directional preferences of night passerine migrants. Ring 17, 1-2: 97-116.

Busse P. 2000. Bird station manual. SE European Bird Migration Network, Univ. of Gdańsk. Gdańsk. pp. 264. 
Busse P., Meissner W. 2015. Bird Ringing Station Manual. - De Gruyter Open. Warszawa/Berlin, pp. 211.

Svensson L. 1992. Identification guide to European Passerines. Stockholm, pp. 368. 